\title{
Conformal Geometry and Brain Flattening
}

\author{
Sigurd Angenent ${ }^{1}$, Steven Haker ${ }^{2}$, Allen Tannenbaum ${ }^{2}$, and Ron Kikinis ${ }^{3}$ \\ 1 Department of Mathematics \\ University of Wisconsin, Madison, Wisconsin 53705 \\ 2 Department of Electrical and Computer Engineering \\ University of Minnesota, Minneapolis, MN 55455 \\ tannenba@ece.umn.edu \\ 3 Harvard Medical School, Brigham and Women's Hospital \\ Harvard University, Boston, MA 02115
}

\begin{abstract}
In this paper, using certain conformal mappings from complex function theory, we give an explicit method for flattening the brain surface in a way which is bijective and which preserves angles. The conformal equivalence arises as the solution of a certain elliptic equation on the surface. Then from a triangulated surface representation of the cortex, we indicate how the procedure may be implemented using finite elements. Further, we show how the geometry of the cortical surface and gray/white matter boundary may be studied using this approach. Hence the mapping can be used to obtain an atlas of the brain surface in a natural manner.
\end{abstract}

Keywords: Brain flattening, conformal maps, functional MRI, segmentation.

\section{Introduction}

The problem of flattening or unfolding a highly undulated surface is of major importance for a number of problems in 3D medical visualization. Recently a number of techniques have been proposed to obtain a flattened representation of the cortical surface; see, e.g., $[5,6,7,15,20]$ and the references therein. Flattening the brain surface has uses in many areas including functional magnetic resonance imaging, in which it is important to visualize neural activity within the three dimensional folds of the brain, as well as the study of various types of brain pathology.

Our approach to flattening the brain surface is based on the use of a certain fact from the theory of Riemann surfaces, specifically, that a surface without any handles, holes or self-intersections can be mapped conformally onto the sphere, and any local portion thereof onto a disc. This mapping, known as a conformal equivalence, is conformal in the sense that angles are preserved. It is also bijective (onto and one-to-one) and thus there is no problem with triangles "flipping" or overlapping, and no cuts need be made on the surface.

We should note that our approach is quite different from the previous works cited above in brain flattening which typically consider locally area or length 
preserving deformations. For example, in the nice approaches of $[6,15]$, the authors fit a parameterized deformable surface whose topology is mappable to a sphere. Then, it is possible to represent the brain surface on a planar map by using spherical coordinates.

In our work, the key observation is that the flattening function may be obtained as the solution of a second order elliptic partial differential equation (PDE) on the surface to be flattened. For triangulated surfaces, there exist powerful, reliable finite element procedures which can be employed to numerically approximate the flattening function.

\section{Flattening the Brain Surface}

In this section, we sketch the mathematical justification of our brain flattening procedure. Full details appear in [1]. A basic assumption is that the brain surface is topologically a sphere. While this is is not exactly correct (there are some small holes where the ventricles connect to the outer surface), we can always fill these in by using, e.g., morphological dilation and erosion. This will not affect the structures in which we are interested in flattening, in particular the brain hemispheres. Let $\Sigma \subset \mathbf{R}^{3}$ represent this brain model which we assume is an embedded surface (no self-intersections) of genus zero. In this section, since we will be giving the analytical solution to the uniformization problem, we assume that $\Sigma$ is a smooth manifold. For the finite element method described in the next section, it will be enough to take it as a triangulated surface. (We refer the reader to [10] for the basic theory of uniformization of Riemann surfaces, and to [16] for the solutions of elliptic PDE's and the Dirichlet problem.) Fix a point $p$ on this surface. Let $\delta_{p}$ denote the Dirac delta (impulse) function at $p$, $\Delta$ the Laplace-Beltrami operator on $\Sigma \backslash\{p\}$, and $i$ the square root of -1 . The Laplace-Beltrami operator is the generalization of the usual Laplacian operator to a smooth surface. Let $S^{2}$ denote the unit sphere in $\mathbf{R}^{3}$ and let $\mathbf{C}$ be the complex plane.

The following result provides the analytical basis for our brain mapping procedure:

A conformal equivalence $z: \Sigma \backslash\{p\} \rightarrow S^{2} \backslash\{$ north pole $\}$ may be obtained by solving the equation

$$
\Delta z=\left(\frac{\partial}{\partial u}-i \frac{\partial}{\partial v}\right) \delta_{p} .
$$

Here, $u$ and $v$ are conformal coordinates defined in a neighborhood of $p$. Further, we are identifying $S^{2} \backslash$ north pole\} with the complex plane in the standard way from complex analysis, say via stereographic projection. This result means that we can get the conformal equivalence by solving a second order partial differential equation on the surface. Fortunately, on a triangulated surface, this may be carried out using a finite element technique we will describe below. 


\section{$3 \quad$ Finite Element Approximation of Conformal Mapping}

We now describe a numerical procedure for solving (1), assuming that $\Sigma$ is a triangulated surface. Using the notation of the previous section, let $\sigma=A B C$ be the triangle in whose interior the point $p$ lies.

\subsection{Functional Interpretation}

The first step in the solution of (1) is to interpret $\left(\frac{\partial}{\partial u}-i \frac{\partial}{\partial v}\right) \delta_{p}$ as a functional on an appropriate space of functions, in our case the finite-dimensional space $P L(\Sigma)$ of piecewise linear functions on $\Sigma$. For any function $f$ smooth in a neighborhood of $p$, one has

$$
\iint_{\Sigma} f\left(\frac{\partial}{\partial u}-i \frac{\partial}{\partial v}\right) \delta_{p} d S=-\left.\left(\frac{\partial f}{\partial u}-i \frac{\partial f}{\partial v}\right)\right|_{p},
$$

and for $f \in P L(\Sigma)$, this last quantity is completely determined by the value of $f$ at $A, B$, and $C$.

Choose the $u$ and the $v$ axes so that $A$ and $B$ are along the $u$ axis, and the positive $v$ axis points towards $C$. Let $E$ be the orthogonal projection of $C$ on $A B$. Then for $f \in P L(\Sigma)$, and $\theta=\frac{\langle C-A, B-A,\rangle}{\|B-A\|^{2}}$, we have

$$
\iint_{\Sigma} f\left(\frac{\partial}{\partial u}-i \frac{\partial}{\partial v}\right) \delta_{p} d S=\frac{f_{A}}{\|B-A\|}-\frac{f_{B}}{\|B-A\|}+i \frac{f_{C}-\left(f_{A}+\theta\left(f_{B}-f_{A}\right)\right)}{\|C-E\|} .
$$

\section{$3.2 \quad$ Finite Elements}

We briefly outline the finite element method for finding our approximation to $z$. The heart of the method simply involves the solution of a system of linear equations [12].

One may show that $z$ satisfies (1) if and only if for all smooth test functions $f$, we have

$$
\iint_{\Sigma} \nabla z \cdot \nabla f d S=\left.\left(\frac{\partial f}{\partial u}-i \frac{\partial f}{\partial v}\right)\right|_{p}
$$

This formulation is the key to the finite element approximation of the solution to (1) on the triangulated surface $\Sigma$. We restrict our attention to $P L(\Sigma)$, and seek a $z \in P L(\Sigma)$ such that (2) holds for all $f \in P L(\Sigma)$.

For each vertex $P \in \Sigma$, let $\phi_{P}$ be the continuous function which is linear on each triangle, has the value 1 at $P$, and is zero at all other vertices. Then these $\phi_{P}$ form a basis for $P L(\Sigma)$, and we seek a $z$ of the form $z=\sum z_{P} \phi_{P}$, for some vector of complex numbers $\left(z_{P}\right), P \in \Sigma$. Further, since (2) is linear in $f$, it is enough to show that (2) holds whenever $f=\phi_{Q}$ for some $Q$.

Thus we want to find a vector $z=\left(z_{P}\right)$ such that for all $Q$,

$$
\sum_{P} z_{P} \iint \nabla \phi_{P} \cdot \nabla \phi_{Q} d S=\frac{\partial \phi_{Q}}{\partial u}(p)-i \frac{\partial \phi_{Q}}{\partial v}(p)
$$




\subsection{Formulation in Matrix Terms}

The formulation (3) is simply a system of linear equations in the complex unknowns $z_{P}$. Accordingly, we introduce the matrix $\left(D_{P Q}\right)$, where

$$
D_{P Q}=\iint \nabla \phi_{P} \cdot \nabla \phi_{Q} d S
$$

for each pair of vertices $P, Q$. Note that $D_{P Q} \neq 0$ only if $P$ and $Q$ are connected by some edge in the triangulation. Thus the matrix $D$ is sparse.

Suppose $P Q$ is an edge belonging to two triangles, $P Q R$, and $P Q S$. A formula from finite-element theory [12], easily verified with basic calculus, says that

$$
D_{P Q}=-\frac{1}{2}\{\cot \angle R+\cot \angle S\}, \quad P \neq Q,
$$

where $\angle R$ is the angle at the vertex $R$ in the triangle $P Q R$, and $\angle S$ is the angle at the vertex $S$ in the triangle $P Q S$. The formula for the diagonal elements of $D$ is (see [1])

$$
D_{P P}=-\sum_{P \neq Q} D_{P Q}
$$

Introducing vectors $a=\left(a_{Q}\right)=\left(\frac{\partial \phi_{Q}}{\partial u}(p)\right)$ and $b=\left(b_{Q}\right)=\left(\frac{\partial \phi_{Q}}{\partial v}(p)\right)$, equation (3), becomes, in matrix terms,

$$
D x=a, \quad D y=-b,
$$

where, using our formula for $\left(\frac{\partial}{\partial u}-i \frac{\partial}{\partial v}\right) \delta_{p}$ derived in Section 3.1, we have

$$
a_{Q}-i b_{Q}:=\left\{\begin{array}{lr}
0 & Q \notin\{A, B, C\}, \\
\frac{-1}{\|B-A\|}+i \frac{1-\theta}{\|C-E\|} & Q=A, \\
\frac{1}{\|B-A\|}+i \frac{\theta}{\|C-E\|} & Q=B, \\
i \frac{-1}{\|C-E\|} & Q=C .
\end{array}\right.
$$

\section{Experimental Results}

We tested our algorithm by flattening the brain surface contained in a $256 \times 256 \times$ 124 MR brain image provided by the Surgical Planning Laboratory of Brigham and Women's Hospital in Boston. These consist of sagittal T1 weighted gradient echo images of a patient with a brain tumor. We chose a brain with a tumor to illustrate the effect of the flattening on both normal and pathological features in an MR brain set.

First, using the segmentation algorithm of $[4,14,18]$, we found the brain cortical surface, i.e the gray matter/CSF interface. The VTK Toolkit [17] was then used to obtain a triangularization of the surface, which we proceeded to smooth slightly to reduce the effects of aliasing. This was done by using the flow according to mean curvature. A morphological based method was used to remove any minute handles on the surface formed by the segmentation process. 
We then used the method described in the previous sections to find a flattening map to the plane and then composed this map with a map from the plane to the unit sphere using standard inverse stereographic projection. This composition gives us a bijective conformal map from the surface to the sphere.

Note that it is not practical to view the planar mapping directly in its entirety, because stereographic projection stretches areas near the north pole too much to be useful. In fact it is not possible to map a sphere, a nearly complete sphere, or any other similarly shaped surface to the plane in any way without major distortion. However, smaller surface patches may be mapped to the plane with a more reasonable amount of distortion, and in fact the "best" (in terms of length distortion) mapping to the plane from a sphere with a geodesic disk removed is known. In practice, we have not found the distortion of area near the north pole to be a problem in solving the linear equations for our flattening map. The method seems to be stable across a wide variety of surface shapes and varying fineness of triangulations.

After flattening the brain surface, we used mean curvature to color corresponding points on the two surfaces (the lighter the point the higher the mean curvature on the brain surface). This provided us with an effective way to see how the flattening process acted on the gyral lines of the brain surface. This is shown in Figure 2, which provides two views of the cortical surface and the corresponding areas on the sphere. Note the tumor on the right parietal lobe visible in the vertex view. It is interesting to see how the conformality of the mapping from the brain surface to the sphere results in a flattened image which is locally very similar in appearance to the original.

Next, we tested our process on the more highly convoluted surface which is defined by the boundary between the white and gray matter within the brain. To extract this boundary, we used a combination of the method based on smoothing posterior probabilities as described in [19], and the segmentation method described in $[4,14,18]$. (See also $[13,21,22]$, and the references therein for other approaches to brain segmentation.) Once the surface was obtained, our flattening method was applied exactly as it was for the cortical surface. The result of this process is shown in Figure 3. Note that much of the white matter surface is hidden within its deep convolutions, but that such areas on the sphere are clearly visible.

We point out that inverting the flattening map allows us easily to establish orthogonal coordinates on the surface as is seen in Figure 4. Further, the method allows us to find north and south poles on a highly convoluted surface such as the brain, giving an alternative method to that discussed in [3].

\section{Conclusions}

In this paper, we described a general method based on a discretization of the Laplace-Beltrami operator for flattening a surface in a manner which preserves the local geometry. The approach can be carried out using a finite element method which takes into account the special boundary conditions. We also il- 
lustrated the technique on the brain surface and white matter of an MR brain data set.

In addition to flattening the cortical surface, we have several other applications in mind including 3D colon and bladder flattening, automatic texture mappings, and image registration. We are very hopeful that our techniques will be useful for such problems as well.

\section{References}

1. S. Angenent, S. Haker, A. Tannenbaum, and R. Kikinis, "Laplace-Beltrami operator and surface unfolding," submitted to IEEE Trans. on Medical Imaging. 272, 274

2. S. Angenent, S. Haker, A. Tannenbaum, and R. Kikinis, "On area preserving mappings of surfaces with minimal distortion," in prepartion.

3. C. Brechbühler, G. Gerig, and O. Kübler, "Parametrization of closed surfaces for 3D shape description," Technical Report, Communication Technology Laboratory, ETH, Zürich, Switzerland, 1996. 275

4. V. Caselles, R. Kimmel, and G. Sapiro, , "Geodesic snakes," Int. Journal of Computer Vision, 1997. 274, 275

5. A. Dale and M. Sereno, "Improved localization of cortical activity by combining EEG and MEG with MRI cortical surface reconstruction: a linear approach," Journal of Cognitive Neuroscience 5 (1993), pp. 162-176. 271

6. C. Davatzikos and R. N. Bryan, "Using a deformable surface model to obtain a shape representation of the cortex," IEEE Transactions on Medical Imaging, 15, (1996), pp. 785-795. 271, 272

7. H. Drury, D. van Essen, C. Anderson, C. Lee, T. Coogan, and J. Lewis, "Computerized mappings of the cerebral cortex: a multiresolution flattening method and a surface-based coordinate system," Journal of Cognitive Neuroscience 8 (1996), pp. 1-28. 271

8. M. P. Do Carmo, Differential Geometry of Curves and Surfaces, Prentice-Hall, Inc., New Jersey, 1976.

9. S. Haker, S. Angenent, A. Tannenbaum, R. Kikinis, G. Sapiro, and M. Halle, "Conformal surface parametrization for texture mappings," Technical Report, Department of Electrical and Computer Engineering, University of Minnesota, January 1999.

10. H. Farkas and I. Kra, Riemann Surfaces, Springer-Verlag, New York 1991. 272

11. I. Hollander, "Cerebral cartography - A method for visualizing cortical structures," Computerized Medical Imaging and Graphics, 19, (1995), pp. 397-415.

12. T. Hughes, The Finite Element Method, Prentice-Hall, New Jersey, 1987. 273, 274

13. T. Kapur, W. Grimson, W. Wells III, and R. Kikinis, "Segmentation of brain tissue from magnetic resonance images," Medical Image Analysis 1 (1996), pp. 109-127. 275

14. S. Kichenasamy, A. Kumar, P. Olver, A. Tannenbaum, A. Yezzi, "Conformal curvature flows: from phase transitions to active contours," Archive Rational Mechanics and Analysis 134 (1996), pp. 275-301. 274, 275

15. D. MacDonald, D. D. Avis, A. C. and Evans, "Multiple surface identification and matching in magnetic resonance images," in Visualization in Biomedical Computing, edited by R. Robb, SPIE Publications, vol. 2359, 1994, pp. 160-169. 271, 272 
16. J. Rauch, Partial Differential Equations, Springer-Verlag, New York 1991. 272

17. W. Schroeder, H. Martin, B. Lorensen, The Visualization Toolkit, Prentice-Hall, New Jersey, 1996. 274

18. K. Siddiqi, A. Tannenbaum, and S. Zucker, "Area and length minimizing flows for image segmentation," IEEE Trans. Image Processing 7 (1998), pp. 433-444. 274, 275

19. P. Teo, G. Sapiro, and B. A. Wandell, "Creating connected representations of cortical gray matter for functional MRI visualization," IEEE Transactions on Medical Imaging, 1998. 275

20. B. Wandell, S. Engel, and H. Hel-Or, "Creating images of the flattened cortical sheet," Invest. Opth. and Vis. Sci. 36 (S612), 1996. 271

21. C. Xu, D. Pham, and J. Prince, "Reconstruction of the central layer of the human cerebral cortex from MR images," Medical Image Computing and ComputerAssisted Intervention, Springer-Verlag, pp. 481-488, 1998. 275

22. X. Zeng, L. Staib, R. Schultz, and J. Duncan, "Volumetric layer segmentation using coupled surfaces propagation," Computer Vision and Pattern Recognition, pp. 708-715, 1998. 275
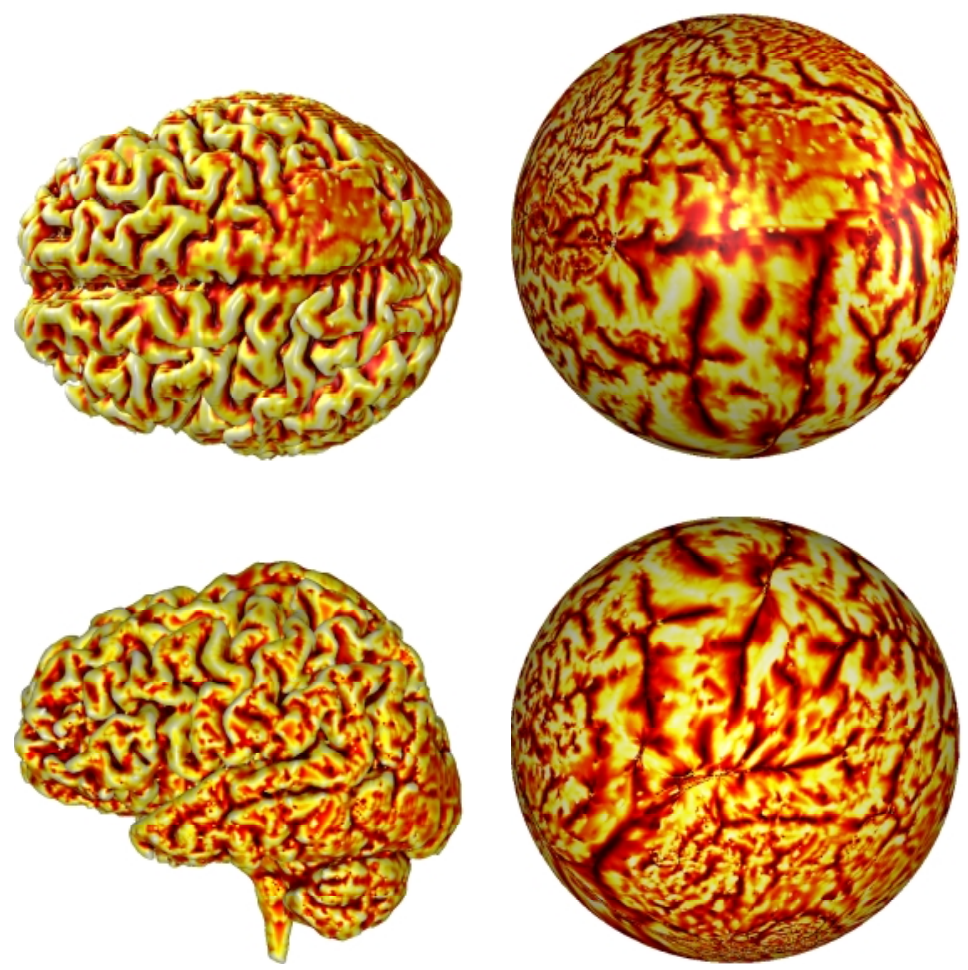

Fig. 1. Two Views of the Flattened Brain Surface 

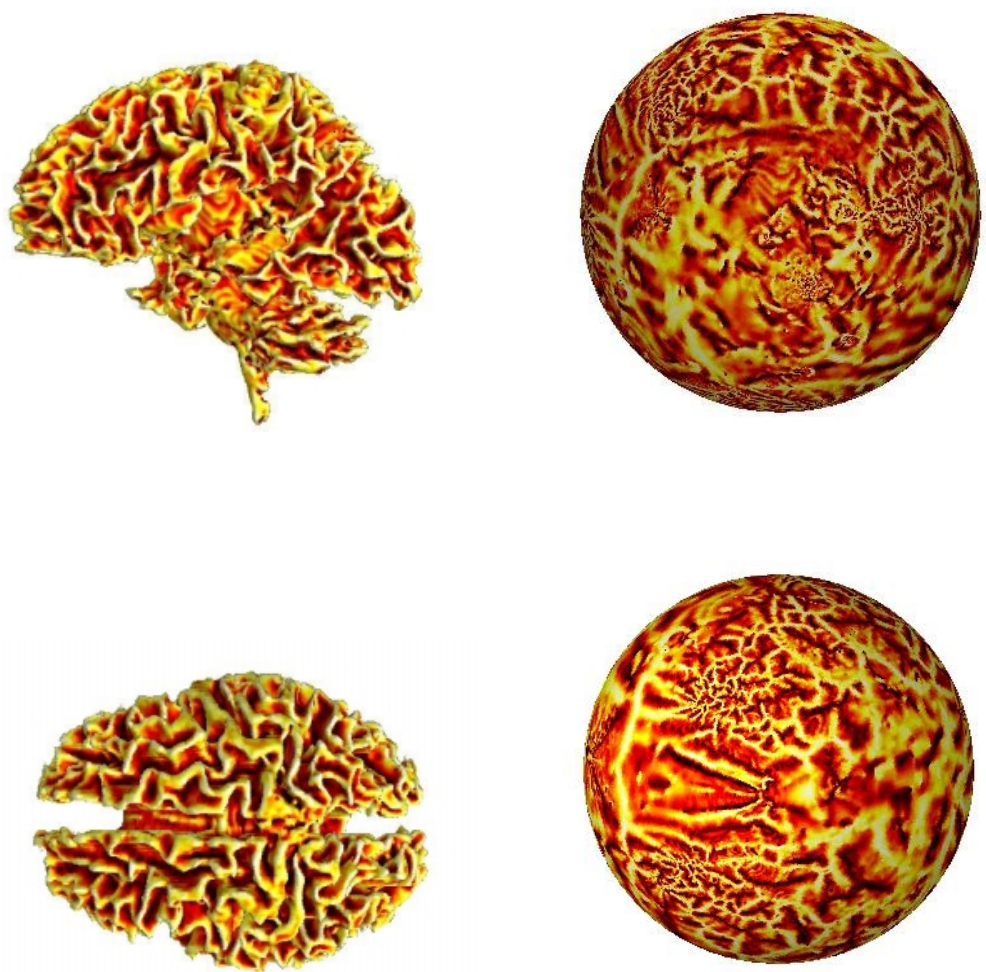

Fig. 2. Two Views of the Flattened White Matter
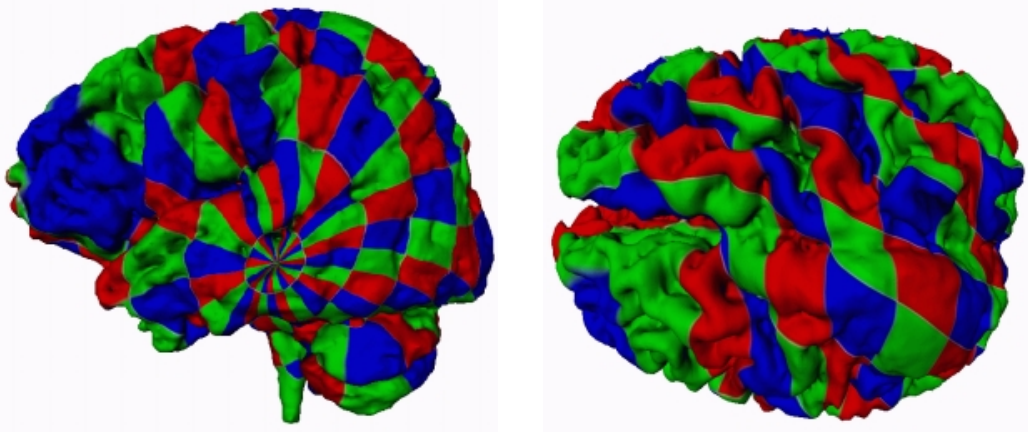

Fig. 3. Orthogonal Grid on Brain Surface 